\title{
Downregulation of UHRF1 promotes EMT via inducing CXCR4 in human cancer cells
}

\author{
YI-DEUN JUNG, JAE-WOONG SHIM, SEONG-JOON PARK, SI HO CHOI, \\ KWANGMO YANG, KYU HEO and MOON-TAEK PARK
}

Research Center, Dongnam Institute of Radiological and Medical Sciences (DIRAMS), Busan 619-953, Republic of Korea

Received October 2, 2014; Accepted December 1, 2014

DOI: $10.3892 /$ ijo.2014.2813

\begin{abstract}
Activation of epithelial-mesenchymal transition (EMT) is important for malignant tumor progression exhibiting migratory and invasive properties. UHRF1 (ubiquitin-like, with PHD and RING finger domains 1), as an epigenetic regulator, plays a crucial role in DNA CpG methylation, chromatin remodeling and gene expression. Many studies demonstrated that UHRF1 is aberrantly expressed in various types of human cancer. However, the precise role of UHRF1 in human cancers remains highly controversial. In the present study, we found that downregulation of UHRF1 enhances the migratory and invasive properties of human cancer cells by inducing EMT, and that the CXCR4 signaling pathway is strictly necessary for UHRF1 deficiency-mediated induction of EMT. Downregulation of UHRF1 induced the expression of the EMT-regulating transcription factors, Zeb1, Slug and Snail and then led to decreased protein level of E-cadherin, and increased protein level of $\mathrm{N}$-cadherin and vimentin, including increased migratory and invasive properties of human cancer cells. In addition, siRNA targeting of Zeb1 or Snail effectively attenuated UHRF1 deficiency-induced EMT, but siRNA targeting of Slug did not, indicating that Zeb1 and Snail play key roles in this event. Moreover, downregulation of UHRF1 induced the expression of CXCR4 in HepG 2 cells. siRNA targeting of CXCR4 greatly suppressed the UHRF1 deficiency-induced EMT, as evidenced by a reversal of expression patterns of Snail and Zeb1, and by reduced migratory and invasive properties of HepG2 cells. In conclusion, our results demonstrate that downregulation of UHRF1 contributes to the induction of EMT in human cancer cells via the activation of
\end{abstract}

Correspondence to: Dr Moon-Taek Park or Dr Kyu Heo, Research Center, Dongnam Institute of Radiological and Medical Sciences (DIRAMS), 40 Jwadong-gil, Jangan-eup, Gijang-gun, Busan 619-953, Republic of Korea

E-mail: mtpark@dirams.re.kr

E-mail:kyuh33@dirams.re.kr

Key words: UHRF1, epithelial-mesenchymal transition, migration, invasion, CXCR4
CXCR4 signaling pathway. Our observation also suggests that UHRF1 may play a pivotal role in suppressing the malignant alteration of cancer cells.

\section{Introduction}

It is well known that the epithelial-mesenchymal transition (EMT) can contribute to motile and invasive properties of cancer cells, which are intimately connected with metastasis (1). During the process of EMT, while the protein levels of E-cadherin (epithelial marker) are decreased, that of $\mathrm{N}$-cadherin and vimentin (mesenchymal markers) are increased (2). The alternations of these proteins have been demonstrated to result in impaired cell-cell adhesion, thereby allowing the spread of cancer cells from the primary sites. Therefore, EMT is mostly thought to lead to tumor progression via promoting cancer cell's ability to invade into the peripheral microenvironment composed of lymph and blood vascular systems (3-5). Many reports have suggested that EMT has a high correlation with the generation of cancer stem cells, the malignancy of tumor cells and the resistance to anticancer drugs $(5,6)$. In addition, EMT has been known to be easily detected at the invasive region of advanced tumors $(7,8)$.

UHRF1 (ubiquitin-like with PHD and RING finger domains 1) was shown to play a pivotal role in maintaining DNA methylation by its binding to hemimethylated DNA for recruiting DNMT1, and correctly imparting the DNA methylation patterns of mother cells to daughter cells (9). Therefore, UHRF1 is well known as an epigenetic regulator comprised of multi-domains containing a N-terminal ubiquitin-like domain, a tandem tudor domain, a PHD domain, an SRA domain and a RING finger motif domain (10). Particularly, both a PHD and an SRA domain of UHRF1 recruit and interact with DNMT1 (10). In addition, the SRA domain of UHRF1 recognizes and binds to hemi-methylated DNA and a RING finger motif accords an E3-ubiquitinliagase function to UHRF1 (10). Most importantly, UHRF1 is known to be an E3-ubiquitin-ligase for the degradation of DNMT1 $(11,12)$. Therefore, overexpression of UHRF1 in cells leads to the global DNA hypomethylation, a hallmark of cancer cells, by decreasing the protein stability of DNMT1 (10-12). Actually, the protein level of UHRF1 is more increased in many cancer cell types, including colon, lung, bladder, pancreatic, prostate, cervical and breast cancer 
cell lines than in normal human cells (13). Disruption in forming the PCNA/DNMT1/UHRF1 complex has been also known to cause the global DNA hypomethylation and high frequency of oncogenic transformation. Moreover, the global DNA hypomethylation can also occur via depletion of UHRF1 $(14,15)$. However, the precise role of UHRF1 depletion in the cancer development has not been clarified.

In the present study, we investigated the role of downregulation of UHRF1 in EMT concerned with tumor malignancy, and found that downregulation of UHRF1 contributes to enhanced EMT of human cancer cells via increasing the expression level of CXCR4. Our observation also suggests that UHRF1 depletion may play a pivotal role in inducing the malignant alteration of cancer cells.

\section{Materials and methods}

Reagents. A mouse monoclonal antibody against UHRF1 (ICBP 90) was purchased from BD Transduction Laboratories (San Jose, CA, USA). Mouse monoclonal antibodies against vimentin, E-cadherin, N-cadherin, Zeb1, Slug, Snail and Twist were purchased from Santa Cruz Biotechnology (Santa Cruz, CA, USA). Mouse monoclonal anti- $\beta$-actin antibody, horseradish peroxidase-conjugated anti-rabbit IgG, and anti-mouse IgG antibodies were purchased from SigmaAldrich Co. (St. Louis, MO, USA). APC-conjugated mouse monoclonal anti-CD44 antibody, PerCP-Cy5.5-conjugated mouse monoclonal anti-CD117 antibody, PE-Cy7-conjugated mouse monoclonal anti-CD184 antibody, FITC-conjugated mouse monoclonal antibodies against CD24, CD47, CD90 and CD340, and phycoerythrin (PE)-conjugated mouse monoclonal anti-CD126 antibody were purchased from BD Transduction Laboratories. PE-conjugated mouse monoclonal antibodies against CD133 and CD326 were purchased from Miltenyi Biotec Inc. (Auburn, CA, USA).

Cell culture. Human hepatocellular carcinoma HepG2 and Hep3B cells, human breast cancer MDA-MB-231 and MCF-7 cells, and human colon cancer HCT116 cells were from the American Type Culture Collection (ATCC, Manassas, VA, USA). Cells were cultured in a humidified $5 \% \mathrm{CO}_{2}$ atmosphere at $37^{\circ} \mathrm{C}$. HepG2 and Hep3B cells were maintained in Dulbecco's modified Eagle's medium (Sigma-Aldrich) supplemented with $10 \%$ (v/v) bovine calf serum, penicillin (50 units/ml) and streptomycin $(50 \mu \mathrm{g} /$ ml) (all from Gibco-BRL, Grand Island, NY, USA). MDA-MB-231 and MCF-7 cells were maintained in DMEM supplemented with $10 \%$ (v/v) bovine calf serum, penicillin (50 units $/ \mathrm{ml})$ and streptomycin $(50 \mu \mathrm{g} / \mathrm{ml})$. HCT116 cells were maintained in McCoy's 5A medium (Gibco-BRL) with $10 \%(\mathrm{v} / \mathrm{v})$ bovine calf serum, penicillin (50 units $/ \mathrm{ml}$ ) and streptomycin $(50 \mu \mathrm{g} / \mathrm{ml})$.

Establishment of inducible UHRF1 shRNA-containing cell lines. The appropriate double-stranded oligonucleotide (5'-CCG GCC GCA CCA AGG AAT GTA CCA TCT CGA GAT GGT ACA TTC CTT GGT GCG GTT TTT-3' and its complement) was cloned into the pLKO.1-puro-shRNA vector (Sigma-Aldrich). HepG2 cells were transfected with the plasmid or control empty vector, using Lipofectamine 2000
(Gibco-Invitrogen Corp., Carlsbad, CA, USA) in accordance with the manufacturer's recommendations. UHRF1 shRNAcontaining stable clones were selected using $1 \mu \mathrm{g} / \mathrm{ml}$ puromycin (Sigma-Aldrich). Stable clones were isolated and endogenous UHRF1 knockdown was determined by western blot analysis using an anti-UHRF1 antibody.

Small interfering RNA transfection. RNA interference mediated by siRNAs was achieved using double-stranded RNA molecules. The siRNAs against UHRF1, Zeb1, Slug, Snail or CXCR4 were purchased from Santa Cruz Biotechnology. Control siRNA-A (Santa Cruz Biotechnology) was used as a control. Cells were grown to $30 \%$ confluency on $60-\mathrm{mm}$ dishes and transfected with the siRNA duplexes (100 nM), using Lipofectamine 2000 (Gibco-Invitrogen) in accordance with the manufacturer's instructions. Assays were performed $48 \mathrm{~h}$ after transfection.

Western blot analysis. Cells were treated with lysis buffer [40 mM Tris- $\mathrm{HCl}$ (pH 8.0), $120 \mathrm{mM} \mathrm{NaCl}$ and $0.1 \%$ (v/v) NP40] supplemented with protease inhibitor cocktail tablets (1 tablet/50 ml; Boehringer, Mannheim, Germany) and centrifuged for $15 \mathrm{~min}$ at $12,000 \mathrm{x} \mathrm{g}$ at $4^{\circ} \mathrm{C}$. Proteins $(30 \mu \mathrm{g})$ were separated by SDS-PAGE and transferred to nitrocellulose membranes (Bio-Rad Laboratories, Hercules, CA, USA). The membranes were blocked with $5 \%$ (w/v) non-fat dry milk in Tris-buffered saline and then incubated for $1 \mathrm{~h}$ with primary antibodies at the dilution of 1:1,000, at room temperature. Specific reaction bands were detected using peroxidase-conjugated secondary antibodies at the dilution of 1:500, and proteins were visualized using an enhanced chemiluminescence system (Amersham Biosciences, Piscataway, NJ, USA).

RNA preparation and RT-PCR (reverse transcriptase-polymerase chain reaction). Total RNA was extracted from the cells using the TRIzol reagent (Invitrogen), and $1 \mu \mathrm{g}$ of the isolated total RNAs was reverse transcribed to complementary DNA (cDNA) using the iScript ${ }^{\mathrm{TM}}$ cDNA synthesis kit (BioRad Laboratories) according to the manufacturer's instructions. The reaction was performed at $25^{\circ} \mathrm{C}$ for $5 \mathrm{~min}$ and then at $42^{\circ} \mathrm{C}$ for $30 \mathrm{~min}$, and was terminated at $85^{\circ} \mathrm{C}$ for $5 \mathrm{~min}$. Next, the cDNA was diluted to $100 \mu \mathrm{l}$, and then $2 \mu \mathrm{l}$ of it was used as a template for RT-PCR. Total reaction volume $(25 \mu \mathrm{l})$ containing $12.5 \mu 1$ of Premix Taq (Takara Bio, Shiga, Japan), $2 \mu 1$ of a template, and $10 \mathrm{pM}$ of both forward and reverse primers was used for PCR amplification which was performed on a C1000 thermal cycler (Bio-Rad Laboratories) using a thermal profile of beginning at $94^{\circ} \mathrm{C}$ for $5 \mathrm{~min}$, followed by 30 cycles at $94^{\circ} \mathrm{C}$ for $10 \mathrm{sec}, 58^{\circ} \mathrm{C}$ for $15 \mathrm{sec}$ and $72^{\circ} \mathrm{C}$ for $30 \mathrm{sec}, 1 \mathrm{cycle}$ of $72^{\circ} \mathrm{C}$ for $10 \mathrm{~min}$ and extention at $12^{\circ} \mathrm{C}$. All primers were designed using the Primer3 program (http://bioinfo.ut.ee/primer3/). The primer used for RT-PCR was as follows: $C X C R 4$ forward (5'-TGC TTG CTG AAT TGG AAG TG-3') and reverse (5'-AGT CAT AGT CCC CTG AGC CC-3'); G3PDH forward (5'-GAA ATC CCA TCA CCA TCT TCC AGG-3') and reverse (5'-GAG CCC CAG CCT TCT CCA TG-3'). Amplified products were separated on $1 \%(\mathrm{w} / \mathrm{v})$ ethidium bromide-stained agarose gels and expression levels were measured by luminescent image analysis. 
Quantitative real-time PCR ( $q R T$ - $P C R$ ). Quantitative reverse transcription-PCR was performed using the SYBR-Green reporter. Total RNA (1 $\mu \mathrm{g})$ isolated using the RNeasy Mini kit (Qiagen, Valencia, CA, USA) was subsequently reverse transcribed to cDNA with the SuperScript First-Strand Synthesis system (Gibco-Invitrogen). cDNA (3 $\mu 1)$ were added to $17 \mu \mathrm{l}$ of the reaction mixture containing $5 \mu \mathrm{l}$ of $\mathrm{ddH}_{2} \mathrm{O}, 10 \mu \mathrm{l}$ of $2 \mathrm{X}$ SYBR-Green Master Mix (Thermo Fisher Scientific, Waltham, MA, USA) and $1 \mu \mathrm{l}$ of each primer. PCR amplification was carried out using a thermal profile of beginning at $94^{\circ} \mathrm{C}$ for $5 \mathrm{~min}$, followed by 40 cycles at $94^{\circ} \mathrm{C}$ for $10 \mathrm{sec}, 58^{\circ} \mathrm{C}$ for $15 \mathrm{sec}$ and $72^{\circ} \mathrm{C}$ for $15 \mathrm{sec}$, and last extension at $72^{\circ} \mathrm{C}$ for $5 \mathrm{~min}$. The amplification specificity of each qRT-PCR analysis was confirmed by the melting curve analysis. The transcripts of target genes were analyzed using a CFX96 ${ }^{\mathrm{TM}}$ Real-Time PCR detection system (Bio-Rad Laboratories). The primers used for qRT-PCR were as follows: Zebl forward (5'-GCA CCT GAA GAG GAC CAG AG-3') and reverse (5'-TGC ATC TGG TGT TCC ATT TT-3'); Slug forward (5'-AGA TGC ATA TTC GGA CCC AC-3') and reverse (5'-CCT CAT GTT TGT GCA GGA GA-3'); Snail forward (5'-GAA AGG CCT TCA ACT GCA AA-3') and reverse (5'-TGA CAT CTG AGT GGG TCT GG-3'); $C X C R 4$ forward (5'-AAA TGG GCT CAG GGG ACT AT-3') and reverse (5'-TCC CAA AGT ACC AGT TTG CC-3'); G3PDH forward (5'-ACC ACA GTC CAT GCC CAT CAC-3') and reverse (5'-TCC ACC ACC CTG TTG CTG TA-3'). G3PDH gene was used as a control to normalize the expression value of Zebl, Slug, Snail or CXCR4. The relative expression level of the genes was calculated using the $2^{-\Delta \Delta \mathrm{Ct}}$ method (16).

Migration and invasion assays. Cell migration and invasion assay were performed using the Transwell chamber $(8-\mu \mathrm{m}$ pore size; Corning Life Sciences, Lowell, MA, USA). Cells $\left(2 \times 10^{4}\right)$ were resuspended in $0.2 \mathrm{ml}$ of serum-free growth medium for both cell migration and invasion assay. For migration assay, the cells were added to the interior of the inserts. Growth medium $(0.8 \mathrm{ml})$ supplemented with $10 \%$ (v/v) bovine calf serum was added to the lower chamber. After incubation for $48 \mathrm{~h}$ at $37{ }^{\circ} \mathrm{C}$, the cells attached to the upper surface of the filter were removed with a cotton swab, and migrated cells on the lower surface of the filter were fixed and stained for 15 min with $0.25 \%$ crystal violet (Sigma-Aldrich), 10\% formaldehyde, and $80 \%$ methanol, and then the inserts were washed five times with $\mathrm{ddH}_{2} \mathrm{O}$ and photographed (magnification, x20). Migrated cells were determined by counting cells in five microscopic fields per well, and the extent of migration was expressed as an average number of cells per microscopic field. Cells were imaged by phase contrast microscopy (Nikon Eclipse 80i; Nikon, Tokyo, Japan). For invasion assay, the cells were added to the interior of the inserts precoated with $10 \mathrm{mg} / \mathrm{ml}$ growth factor-reduced Matrigel (BD Biosciences, Bedford, MA, USA). Growth medium $(0.8 \mathrm{ml})$ supplemented with $10 \%(\mathrm{v} / \mathrm{v})$ bovine calf serum was added to the lower chamber. After incubation for $48 \mathrm{~h}$ at $37^{\circ} \mathrm{C}$, the inserts were processed as described above for the migration assay.

Flow cytometric analysis. Single cells were detached from the culture dishes with trypsin-EDTA, counted, $\sim 1 \times 10^{5}$ cells were centrifuged at 2,000 rpm for $5 \mathrm{~min}$ and then resuspended in PBS containing 5\% FBS and $0.1 \% \mathrm{NaN}_{3}$ (Sigma-Aldrich). The cells were incubated at $4^{\circ} \mathrm{C}$ with APC-conjugated anti-CD44, PerCP-Cy5.5-conjugated anti-CD117, PE-Cy7-conjugated anti-CD184, FITC-conjugated anti-CD24, anti-CD47, anti-CD90, anti-CD340, phycoerythrin (PE)-conjugated anti-CD126, anti-CD133 or anti-CD326 antibody for $1 \mathrm{~h}$. The labeled cells were washed twice with PBS, resuspended in PBS, and then analyzed by flow cytometry, FACSAria ${ }^{\mathrm{TM}}$ (Special order system, BD Biosciences).

Statistical analysis. The data presented are representative of at least three independent experiments. Differences between groups were analyzed using the Student's t-test (SPSS Statistics version 17.0; SPSS, Inc., Chicago, IL, USA). P-values <0.05 were considered significant.

\section{Results}

Downregulation of UHRF1 promotes EMT of human cancer cells. Invasion of cancer cells arises from the loss of cell-cell interaction leading to their migratory properties. Importantly, this event is closely related with EMT (3-6). The high expression level of UHRF1 was shown to be correlated with tumor progression (10-13). Recently, however, disruption of DNMT1/PCNA/UHRF1 complex has been suggested to lead to tumorigenesis, implying that a deficiency in UHRF1 may also be involved in cancer development $(14,15)$. Therefore, we investigated whether downregulation of UHRF1 affects EMT of cancer cells. To fulfill this purpose, we introduced shRNAs targeting UHRF1 into HepG2 or MDA-MB-231 cells by transfection and generated stable cell lines. We first used western blot analysis to measure epithelial and mesenchymal cell markers in these stable cell lines. As shown in Fig. 1A, western blot analyses showed an increase in the expression levels of the mesenchymal cell markers $\mathrm{N}$-cadherin and vimentin in UHRF1 shRNA-expressing HepG2 cells, compared with parental HepG2 cells, although the expression of the epithelial cell marker E-cadherin, did not be change. Consistent with these results, UHRF1 shRNA-expressing HepG2 cells displayed more migratory and invasive properties, compared with parental HepG2 cells (Fig. 1B and C). Moreover, we found that UHRF1 shRNA-expressing MDA-MB-231 cells also shows an increase in the expression levels of $\mathrm{N}$-cadherin and vimentin, a decrease in the expression level of E-cadherin, and displays substantial migratory and invasive properties, compared with parental MDA-MB-231 cells (Fig. 1D-F). Collectively, these results indicate that downregulation of UHRF1 may play a key role in initiating EMT of cancer cells.

To further confirm a possible causal connection between downregulation of UHRF1 and EMT in cancer cells, we transiently transfected HepG2 and MDA-MB-231 cells with siRNAs targeting UHRF1, and then performed western blot analysis, migration and invasion assays. In both HepG2 and MDA-MB-231 cells transiently transfected with siRNAs targeting UHRF1, we obtained similar results to those observed in stable cell lines (Fig. 2A-F). In addition, we also found that the expression levels of vimentin increase in Hep3B, MCF-7 and HCT116 transiently transfected with siRNAs targeting UHRF1 (Fig. 2G). These results collectively indicated that 
A

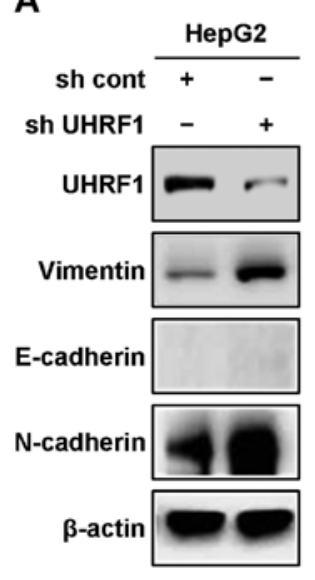

D

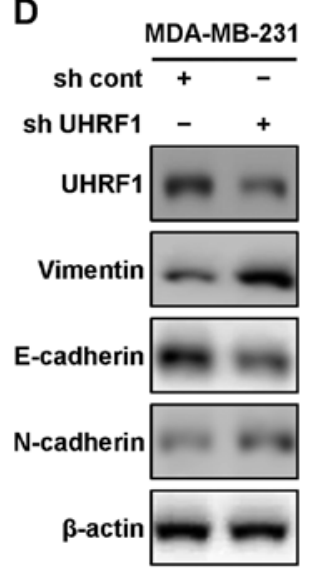

B

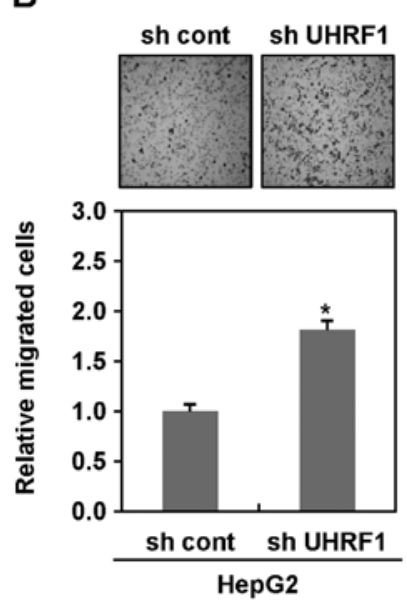

E

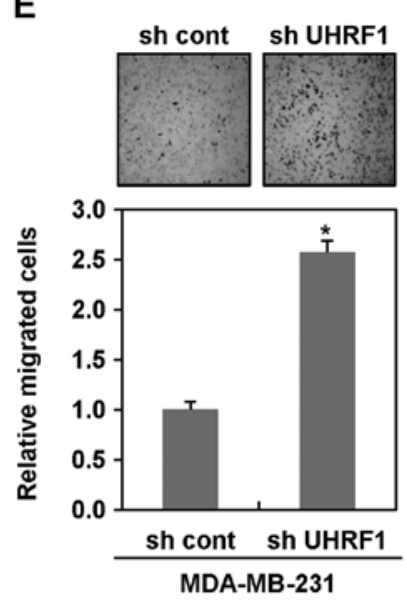

C

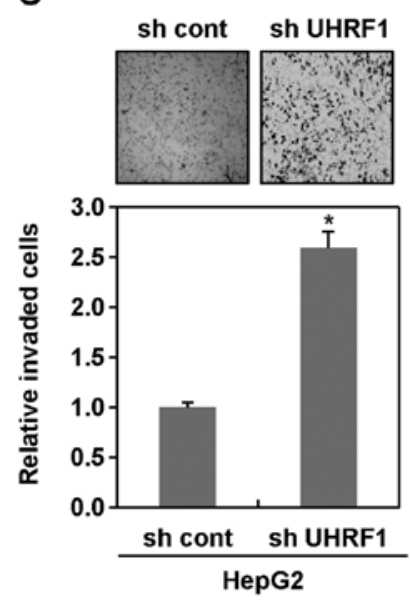

$\mathbf{F}$

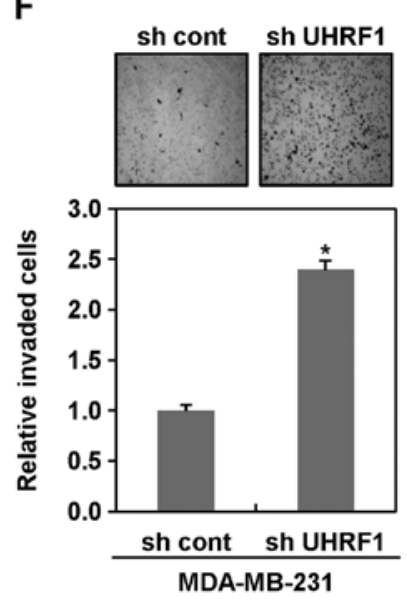

Figure 1. UHRF1-deficient human cancer cells show mesenchymal transformation. (A) Cell lysates from shcont- and shUHRF1 HepG2 cells were subjected to western blot analysis using anti-UHRF1, anti-vimentin, anti-E-cadherin, anti-N-cadherin and anti- $\beta$-actin antibodies. Experiments were conducted in triplicate and the data shown are representative of a typical experiment. (B and C) Increased migratory and invasive properties of UHRF1-deficent HepG2 cells. Migration and invasion assay were performed using the Transwell chamber. Results from three independent experiments are expressed as means \pm SEMs. $\left({ }^{*} \mathrm{P}<0.05\right)$. (D) Cell lysates from shcont- and shUHRF1 MDA-MB-231 cells were subjected to western blot analysis using anti-UHRF1, anti-vimentin, anti-E-cadherin, anti-N-cadherin and anti- $\beta$-actin antibodies. Experiments were conducted in triplicate, and the data shown are representative of a typical experiment. (E and F) Increased migratory and invasive properties of UHRF1-deficent MDA-MB-231 cells. Migration and invasion assay were performed using the Transwell chamber. Results from three independent experiments are expressed as means \pm SEMs $\left({ }^{*} \mathrm{P}<0.05\right)$.

downregulation of UHRF1 clearly contributes to initiating EMT of human cancer cells.

Downregulation of UHRF1 triggers EMT through the induction of Zebl and Snail in human cancer cells. Next, we examined whether downregulation of UHRF1 promotes EMT via EMT-regulating transcription factors, Zeb1, Slug, Snail and Twist in cancer cells. As shown in Fig. 3A and B, we found that Zeb1, Slug and Snail are generally upregulated at the protein level by both shRNAs and siRNAs targeting UHRF1 in HepG2 and MDA-MB-231 cells, whereas there was no change in the protein level of Twist in both cell lines (Fig. 3A and B).

To further determine which of these transcription factors is dominantly involved in EMT caused by downregulation of UHRF1, we transfected HepG2 cells with siRNAs targeting Zeb1, Slug or Snail in presence or absence of UHRF1 siRNAs. Notably, siRNAs targeting Zeb1 or Snail effectively attenuated the expression level of vimentin increased by downregulation of UHRF1, whereas siRNAs targeting Slug did not (Fig. 3C). Furthermore, we found that treatment with siRNAs targeting Zeb1 or Snail efficiently blocks the migratory and invasive properties of HepG2 cells increased by downregulation of UHRF1 (Fig. 3D-I). Taken together, these results suggest that downregulation of UHRF1 promotes EMT through upregulation of Zeb1 and Snail in human cancer cells.

Downregulation of UHRF1 induces the expressions of cancer stem cell-related cell surface markers. As EMT has been suggested to lead to an increase in the population of cancer stem cells $(17,18)$, we next determined the expression of cancer stem cell-related cell surface markers in presence or absence of shRNAs targeting UHRF1 in HepG2 cells. As shown in Fig. 4, flow cytometric data revealed that the cells expressing UHRF shRNAs show an increase in the expressions of CD47, CD90, CD117, CD126, CD133 and CD340, but no significant change in those of CD326, CD24 and CD44. These results 
A

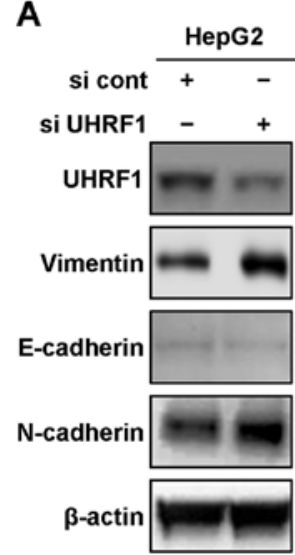

D
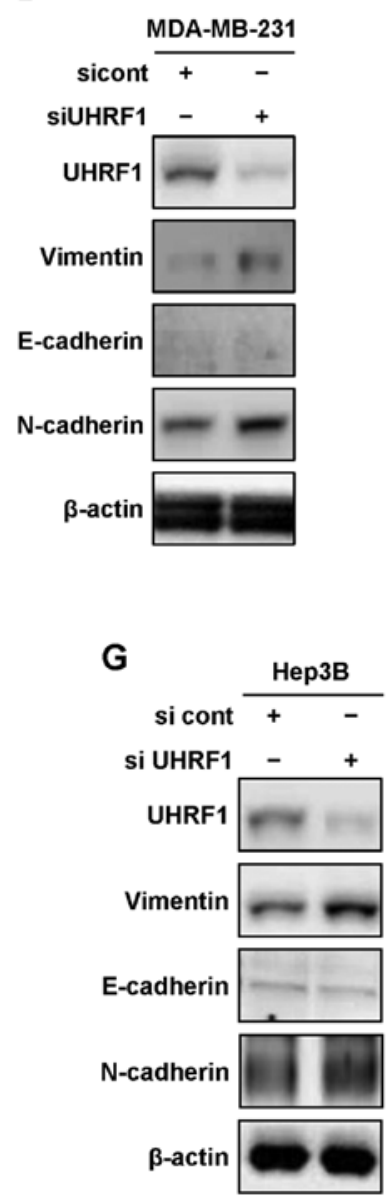

B
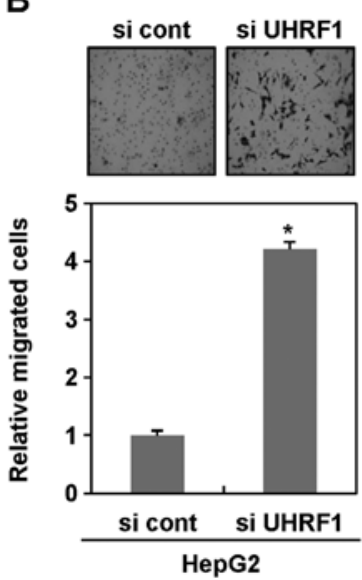

E
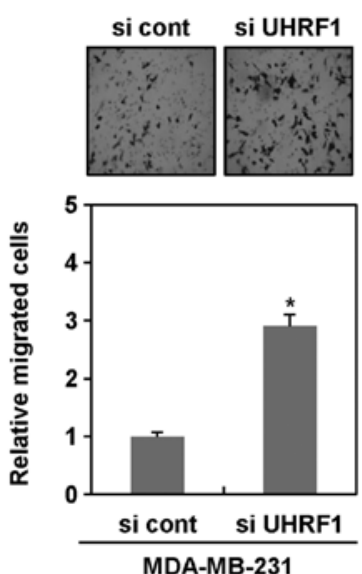

MDA-MB-231
C
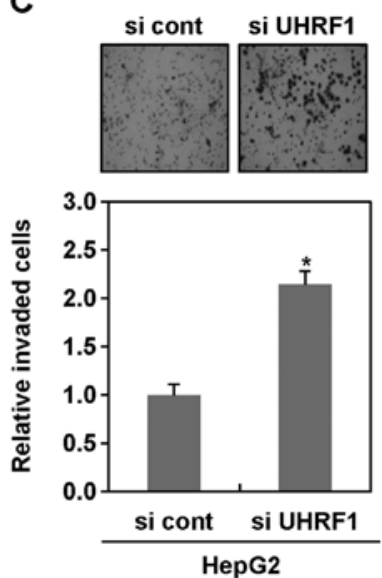

$\mathbf{F}$
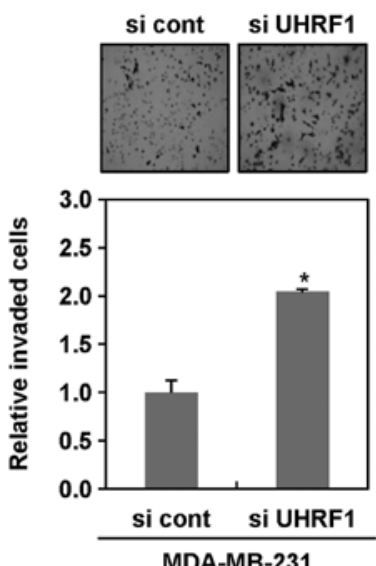

MDA-MB-231

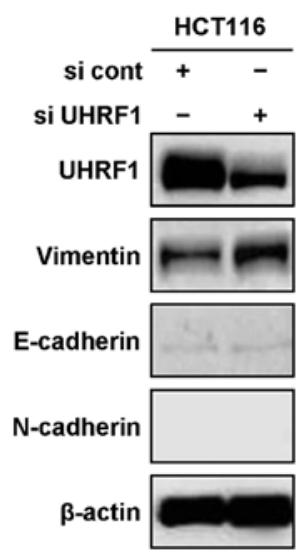

Figure 2. Ectopic downregulation of UHRF1 increases migratory and invasive properties of human cancer cells. (A) After HepG2 cells were grown in presence or absence of siRNA targeting UHRF1 for $48 \mathrm{~h}$, cell lysates were subjected to western blot analysis using anti-UHRF1, anti-vimentin, anti-E-cadherin, anti$\mathrm{N}$-cadherin and anti- $\beta$-actin antibodies. Experiments were conducted in triplicate, and the data shown are representative of a typical experiment. (B and C) Increased migratory and invasive properties of HepG2 cells transiently transfected with siRNA targeting UHRF1. Migration and invasion assay were performed using the Transwell chamber. Results from three independent experiments are expressed as means \pm SEMs. ("P<0.05). (D) After MDA-MB-231 cells were grown in presence or absence of siRNA targeting UHRF1 for $48 \mathrm{~h}$, cell lysates were subjected to western blot analysis using anti-UHRF1, anti-vimentin, anti-E-cadherin, anti-N-cadherin and anti- $\beta$-actin antibodies. Experiments were conducted in triplicate, and the data shown are representative of a typical experiment. (E and F) Increased migratory and invasive properties of MDA-MB-231 cells transiently transfected with siRNA targeting UHRF1. Migration and invasion assay were performed using the Transwell chamber. Results from three independent experiments are expressed as means \pm SEMs. ("P $<0.05$ ). (G) After Hep3B, MCF-7 and HCT116 cells were grown in presence or absence of siRNA targeting UHRF1 for $48 \mathrm{~h}$, cell lysates were subjected to western blot analysis using anti-UHRF1, anti-vimentin, anti-E-cadherin, anti-N-cadherin and anti- $\beta$-actin antibodies. Experiments were conducted in triplicate, and the data shown are representative of a typical experiment.

indicated that downregulation of UHRF1 can increase the expression of cancer stem cell-related cell surface markers, which are thought to be closely associated with the generation of cancer stem cells. 
A

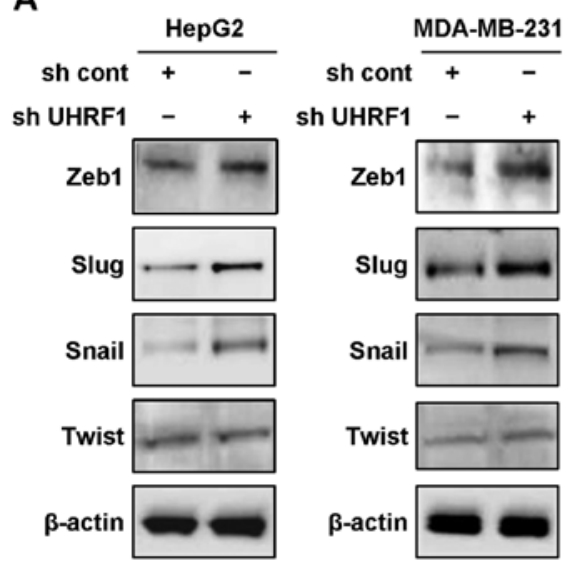

B

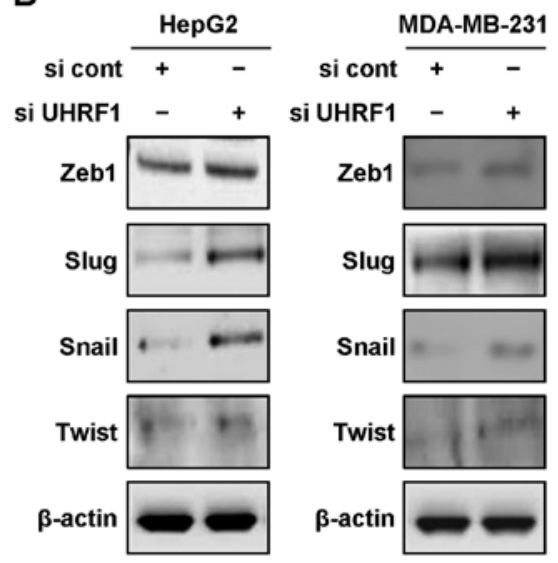

C
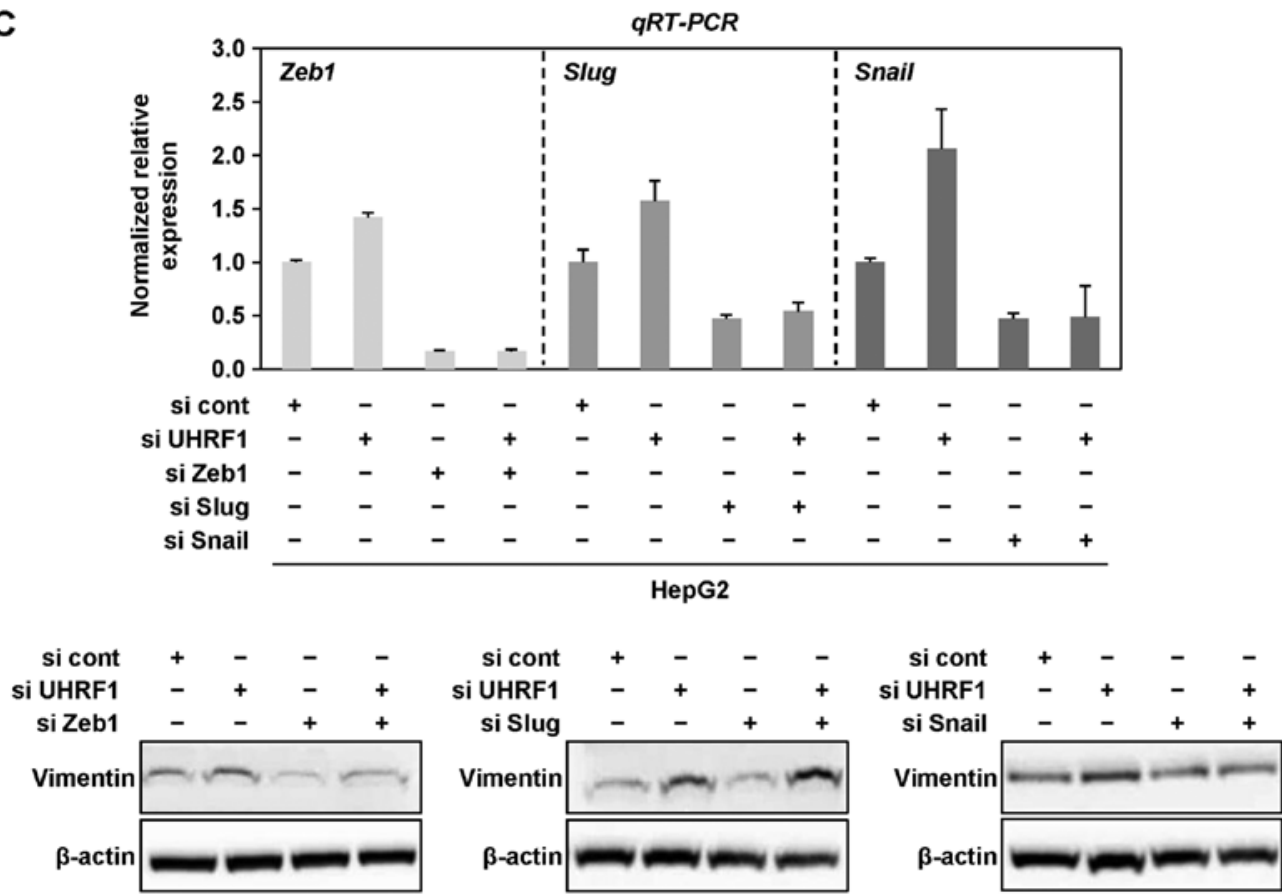

Figure 3. Downregulation of UHRF1 leads to EMT via EMT transcription factors Zeb1 and Snail in human cancer cells. (A) Cell lysates from HepG2 and MDA-MB-231 cells stably transfected with UHRF1 shRNA were subjected to western blot analysis using anti-Zeb1, anti-Slug, anti-Snail, anti-Twist and anti- $\beta$-actin antibodies. Experiments were conducted in triplicate, and the data shown are representative of a typical experiment. (B) After HepG2 and MDA-MB-231 cells were grown in presence or absence of siRNA targeting UHRF1 for $48 \mathrm{~h}$, cell lysates were subjected to western blot analysis using antiZeb1, anti-Slug, anti-Snail, anti-Twist and anti- $\beta$-actin antibodies. Experiments were conducted in triplicate, and the data shown are representative of a typical experiment. (C) After HepG2 cells were transfected with siRNA targeting Zeb1, Slug or Snail in presence or absence of UHRF1 siRNA for 48 h, qRT-PCR was conducted using primers designed for Zeb1, Slug or Snail, and cell lysates were subjected to western blot analysis using anti-vimentin and anti- $\beta$-actin antibodies. Experiments were conducted in triplicate, and the data shown are representative of a typical experiment.

CXCR4 contributes to EMT caused by downregulation of UHRF1. Especially, CD90 (Thy-1), CD117 (c-Kit), CD340 (HER2) and CD184 (CXCR4) increased in HepG2 cells expressing UHRF1 shRNAs are well known to be receptortyrosine kinases and a $\mathrm{G}$ protein-coupled receptor possessing a great ability in a variety of cellular events (19-22). Moreover, among them, CXCR4 was demonstrated to induce an aggressively malignant phenotype of cancer cells via driving EMT $(23,24)$. Therefore, we next examined whether CXCR4 is critically involved in EMT caused by downregulation of UHRF1. To better assess the expression level of CXCR4 in the presence or absence of shRNAs targeting UHRF1 in HepG2 cells, we performed RT and qRT-PCR analysis. As shown in Fig. 5A, mRNA expression of CXCR4 was markedly increased in the cells expressing UHRF1 shRNAs. To further confirm the increase in mRNA expression of CXCR4, we transiently transfected HepG2 cells with siRNAs targeting UHRF1 and performed qRT-PCR analysis. In HepG2 cells transiently transfected with siRNAs targeting UHRF1, we obtained similar results to those observed in stable cell lines, indicating that downregulation of UHRF1 efficiently increases the expression of CXCR4 (Fig. 5B).

We next examined whether CXCR4 contributes to EMT caused by downregulation of UHRF1. As shown in Fig. 5C-E, we found that siRNA-mediated CXCR4 knockdown greatly inhibited the expression of both Snail and Zeb1 increased 
D

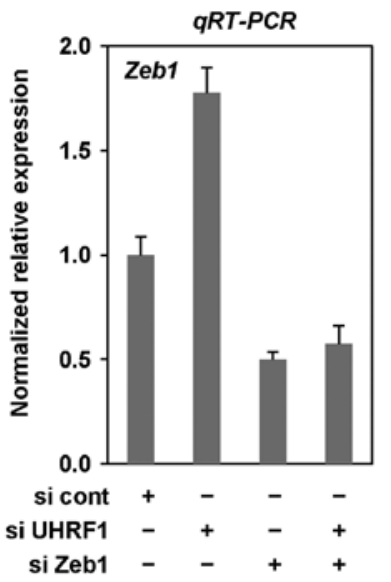

G

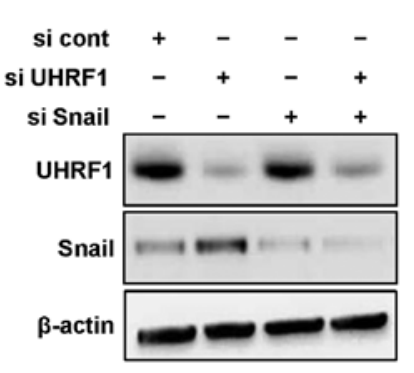

E
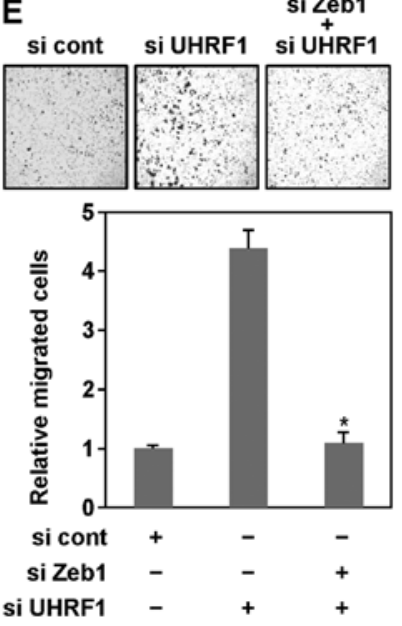

H
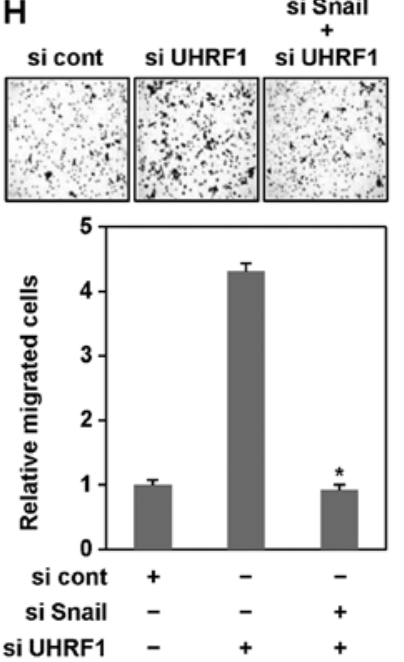

F si Zeb1
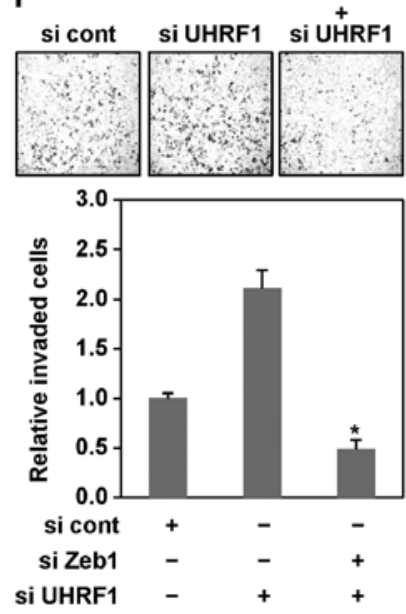

I
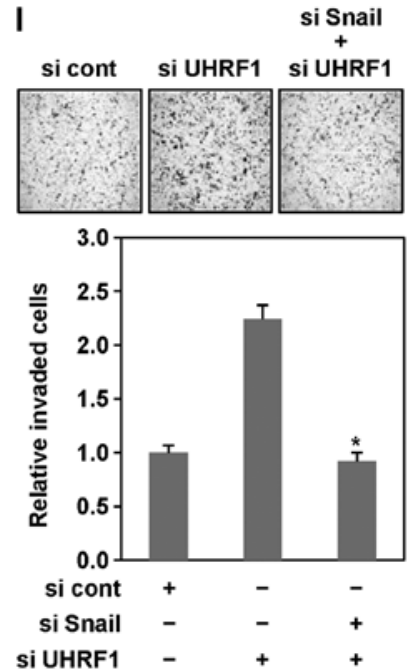

Figure 3. Continued. (D) After HepG2 cells were transfected with siRNA targeting Zeb1 in presence or absence of UHRF1 siRNA for 48 h, qRT-PCR was conducted using primers designed for Zeb1. (E and F) Migration and invasion assay were performed using the Transwell chamber. Results from three independent experiments are expressed as means \pm SEMs. ( $(\mathrm{P}<0.05)$. (G) After HepG2 cells were transfected with siRNA targeting Snail in presence or absence of UHRF1 siRNA for $48 \mathrm{~h}$, cell lysates were subjected to western blot analysis using anti-UHRF1, anti-Snail and anti- $\beta$-actin antibodies. (H and I) Migration and invasion assay were performed using the Transwell chamber. Results from three independent experiments are expressed as means \pm SEMs ("P<0.05).

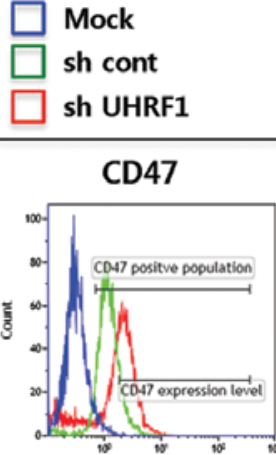

CD184

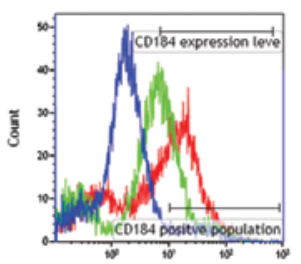

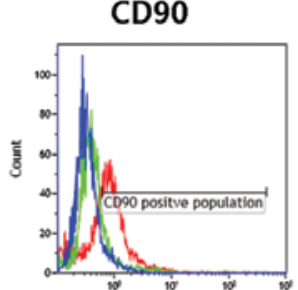

CD340

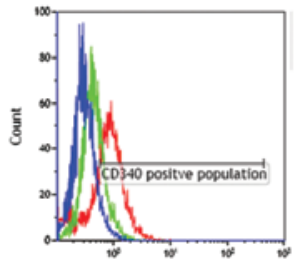

HepG2

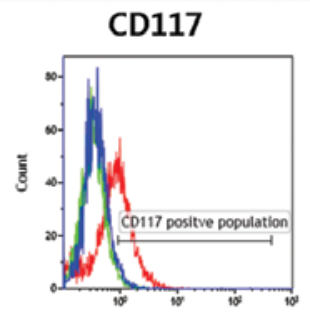

CD326

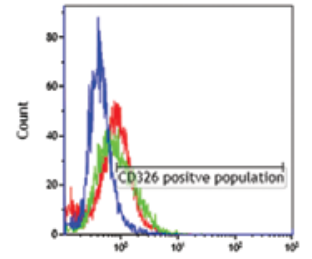

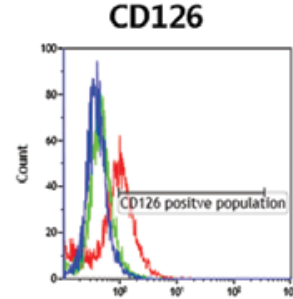

CD24

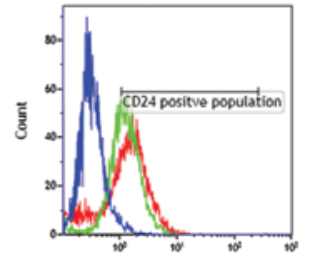

CD133

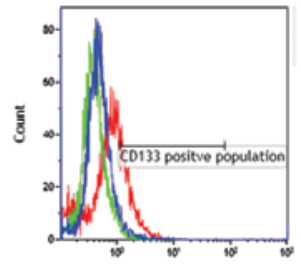

CD44

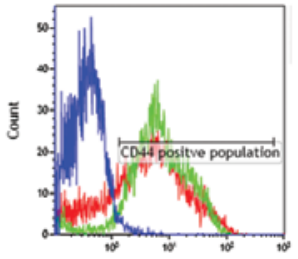

Figure 4. Downregulation of UHRF1 leads to the expression of cell surface markers for cancer stem cells. The shcont- and shUHRF1 HepG2 cells were stained with anti-cell surface marker antibodies, and then the expression levels were measured by flow cytometric analysis. 
A
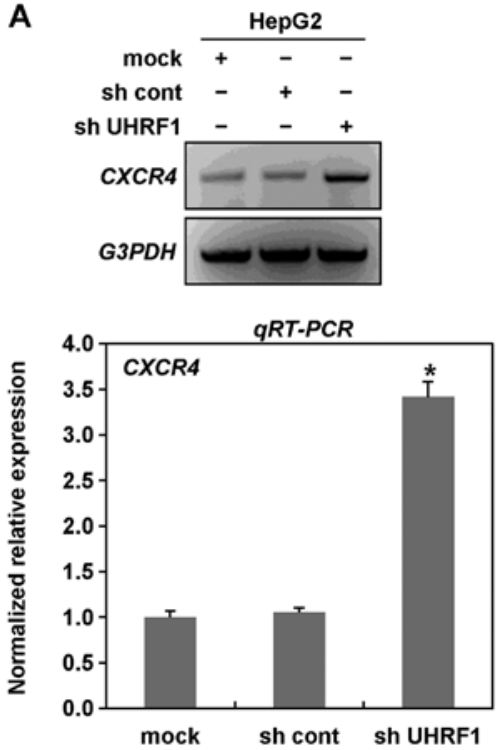

C

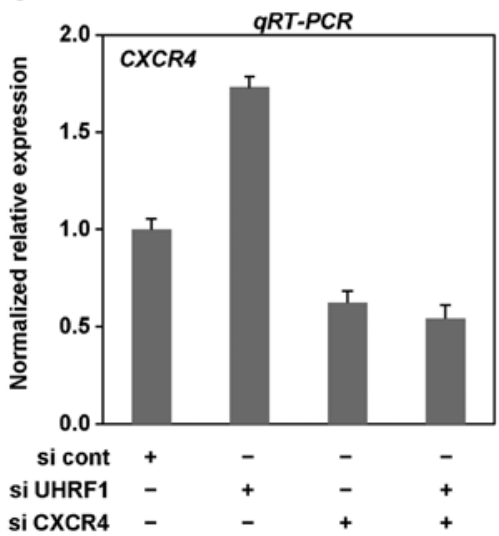

B

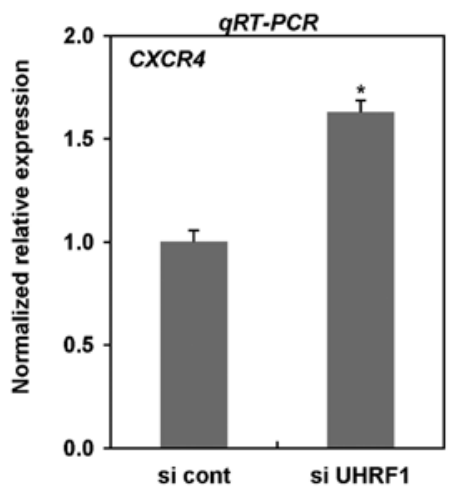

D

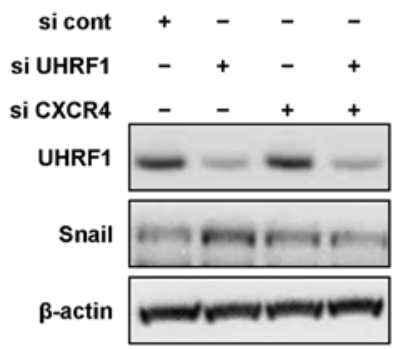

E

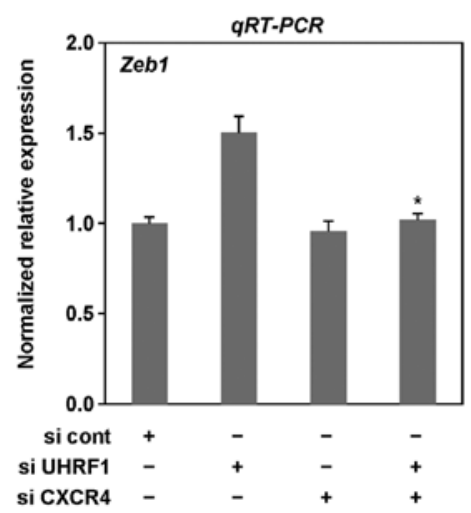

F

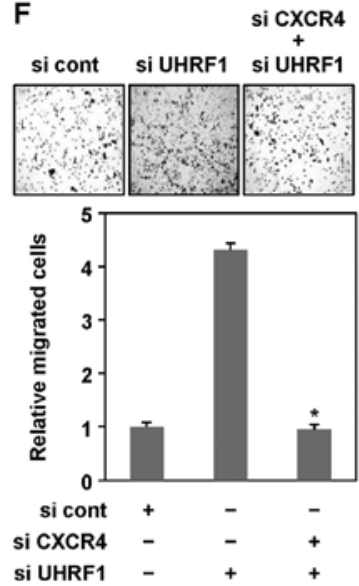

G
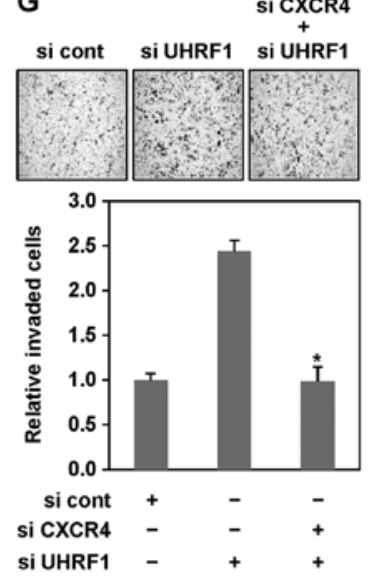

Figure 5. Upregulation of CXCR4 plays a crucial role in the induction of EMT in UHRF1-deficient cancer cells. (A) cDNAs obtained from shcont- and shUHRF1 HepG2 cells were subjected to RT-PCR and qRT-PCR using primers designed for CXCR4. Experiments were conducted in triplicate, and the data shown are representative of a typical experiment. (B) After HepG2 cells were grown in presence or absence of siRNA targeting UHRF1 for 48 h, qRT-PCR was conducted using primers designed for CXCR4. Experiments were conducted in triplicate, and the data shown are representative of a typical experiment. (C and D) After HepG2 cells were transfected with siRNA targeting CXCR4 in presence or absence of UHRF1 siRNA for $48 \mathrm{~h}, \mathrm{qRT}$-PCR was conducted using primers designed for CXCR4, and cell lysates were subjected to western blot analysis using anti-UHRF1, anti-Snail and anti- $\beta$-actin antibodies. Experiments were conducted in triplicate, and the data shown are representative of a typical experiment. (E-G) After HepG2 cells were transfected with siRNA targeting CXCR4 in presence or absence of UHRF1 siRNA for $48 \mathrm{~h}$, qRT-PCR was conducted using primers designed for Zeb1, and migration and invasion assay were performed using the Transwell chamber. Results from three independent experiments are expressed as means \pm SEMs ( $\left.{ }^{*} \mathrm{P}<0.05\right)$.

by downregulation of UHRF1. Moreover, siRNA-mediated CXCR4 knockdown effectively modulated both migratory and invasive properties of HepG2 cells increased by downregulation of UHRF1 (Fig. 5F and G). Collectively, these results 
clearly indicate that CXCR4 plays key roles in EMT induced by downregulation of UHRF1 in human cancer cells.

\section{Discussion}

Recently, EMT defining that epithelial cells acquire mesenchymal characteristics has been implicated to be highly relevant to the acquisition of malignant properties by cancer cells (1,3-6). Especially, EMT confers the early steps of metastasis including local invasion and subsequent spreading of cancer cells to distant locations $(25,26)$. Although, in this context, the epigenetic pathways activating EMT are extensively investigated (26), the epigenetic mechanism for initiating the malignancy of cancer cells is well not understood. In the present study, we found that downregulation of UHRF1, an epigenetic regulator, contributes to the increase in EMT of human cancer cells via inducing the expression of CXCR4.

UHRF1 has been reported to be involved in a variety of physiological and pathological events, from development to cancer progression $(13,27)$. The expression levels of UHRF1 are highly upregulated in diverse cancer types (13). Indeed, overexpression of UHRF1 has been demonstrated to play pivotal roles in malignant cellular transformation via repressing either transcriptional or protein level of tumor suppressors, and inducing the global DNA hypomethylation which leads to genomic instability, a hallmark of cancer cells (10-12). However, the precise mechanism by which UHRF1 inhibits tumor-suppressors has not yet been clarified.

Furthermore, it has been reported that cells overexpressing UHRF1 show increased growth and migration with morphological features resembling those occurring in EMT in colorectal cancer, suggesting that high expression level of UHRF1 may lead to the phenotypic changes of cancer cells by inducing EMT (28). In addition, several reports showed that overexpression of UHRF1 promotes metastasis in colorectal, gastric and breast cancer $(13,29-31)$. Thus, UHRF1 is considered as an oncogene driving tumorigenesis (32).

Since UHRF1 does not only recognize hemimethylated DNA to recruit DNMT1 for methylation on newly synthesized DNA strands, but also has a RING domain with ubiquitin E3 ligase activity, UHRF1 can readily reduce the protein stability of DNMT1 via ubiquitin-proteasome pathway (10-12). Moreover, DNMT1-deficent cells have been reported to show global DNA hypomethylation $(33,34)$. Therefore, the global DNA hypomethylation by upregulation of UHRF1 expression is thought to due to the reduced protein stability of DNMT1.

Recently, the disruption of DNMT1/PCNA/UHRF1 complex has been reported to induce tumorigenesis and the global DNA hypomethylation $(14,15)$. Therefore, it is plausible that UHRF1 deficiency is also associated with further acquisition of invasive properties of cancer cells through disrupting the complex formation. Because both overexpression of and deficiency in UHRF1 affects malignancy of cells, a balanced expression level of UHRF1 is thought to be necessary for the normal behavior of cells. In the present study, we elucidated the possible role of UHRF1 deficiency in the induction of EMT in human cancer cells using sh- or siRNA-mediated knockdown approaches. As expected, we found that both shRNA and siRNA-mediated knockdown of UHRF1 increase the migratory and invasive properties of human cancer cells as well as inducing mesenchymal markers (Figs. 1 and 2), suggesting that UHRF1 deficiency is also responsible for malignancy of human cancer cells. In addition, we observed that downregulation of UHRF1 induces EMT through activating the EMT-regulating transcription factors Zeb1 and Snail, but not Slug and Twist in HepG2 cells (Fig. 3). These transcription factors are thought to cooperatively control both genes involved in epithelial markers, such as E-cadherin, and mesenchymal markers, such as $\mathrm{N}$-cadherin and vimentin via performing dual functions as an activator and a repressor $(35,36)$. Moreover, it has been previously reported that the overexpression of snail can raise the transcriptional level of Zeb1 via activating Zeb1 promoter (37). Consistent with these reports, snail in UHRF1-deficient cancer cells seems to participate in increasing the expression level of Zeb1 to induce EMT. Although the EMT-regulating transcription factors are aberrantly expressed in various cancer types (1), the precise mechanism underlying the regulation of these factors in cancers are not understood.

Emerging evidence suggested that EMT plays a key role not only in metastasis, but also in cancer recurrence well known to be closely associated with generation of cancer stem cells $(17,18)$. Therefore, we investigated whether UHRF1 deficiency affects the expression of cancer stem cell-related surface markers in HepG2 cells. We found that shRNAmediated knockdown of UHRF1 increases the expressions of CD47, CD90, CD117, CD133, CD184 and CD340 in HepG2 cells. These observations indirectly indicate that UHRF1 deficiency may contribute to the generation of cancer stem cells. In addition, since CD117 (c-Kit), CD184 (CXCR4) and CD340 (HER2) are well known to activate intracellular signaling transduction pathways (19-22), there is a possibility that they may lead to EMT in UHRF1-deficient cancer cells.

As one of the best studied chemokine receptors, CXCR4 plays a key role in survival, growth, vasculogenesis, EMT and metastasis in a variety of cancer types $(38,39)$, and is known to interact with its ligand, CXCL12 (also named stromal derived growth factor-1, SDF-1) to activate intracellular signaling pathway (40). Importantly, CXCR4-mediated signaling pathways have been suggested to be crucial for metastatic malignancy (40). In addition, it has been shown that inhibition of CXCR4-mediated pathways by treatment with neutralizing antibodies against, or antisense RNA to CXCR4 greatly attenuates both invasion and migration of cancer cells, and that high expression level of CXCR4 is closely related with metastatic ability of cancer cells in vivo (41). Consistent with these reports, we found that UHRF1 deficiency efficiently increases the expression level of CXCR4 in HepG2 cells in vitro (Figs. 4 and 5). Furthermore, siRNA-mediated knockdown of CXCR4 effectively blocked the expressions of Zeb1 and Snail, and migratory and invasive properties of UHRF1-deficient HepG2 cells (Fig. 5). These observations strongly indicate that CXCR4 contributes to EMT increased in UHRF1-deficient cancer cells. Recent studies also showed that CXCR4 participates in survival and maintenance of cancer stem cells during cancer progression $(42,43)$. In addition, CXCR4 is also deemed a putative cell surface marker 
for cancer stem cells (44). Similarly, because our results indicated that UHRF1 deficiency effectively increases the cancer stem cell-related cell surface markers, as well as CXCR4, further studies are required to elucidate the possible role of UHRF1 deficiency in the generation of cancer stem cells Moreover, a recent study indicates that UHRF1 can recruit and cooperate with G9a, a histone lysine methyltransferase, to suppress the activity of p21 promoter (45). Similarly, as upregulation of CXCR4 by UHRF1 deficiency is thought to be due to disruption of the cooperation between UHRF1 and G9a, additional studies are needed to clarify the suppressive effect of UHRF1 on the induction of CXCR4 gene expression.

In conclusion, we demonstrated that shRNA or siRNA-mediated knockdown of UHRF1 markedly increased EMT in human cancer cells. Furthermore, we present evidence that downregulation of UHRF1 is sufficient to induce EMT-regulating transcription factors Zeb1 and Snail through increasing the expression level of CXCR4, and thereby promotes EMT in human cancer cells. Our observations might provide an important clue as to how malignant tumors develop.

\section{Acknowledgements}

The present research was supported by the National R\&D Program through the Dongnam Institute of Radiological and Medical Sciences (DIRAMS) (50595-2014) and the (Basic Science Research Program) through the National Research Foundation of Korea (NRF) (NRF-2014 M2A2A 7043665) funded by the Ministry of Science, ICT and Future Planning.

\section{References}

1. Tsai JH and Yang J: Epithelial-mesenchymal plasticity in carcinoma metastasis. Genes Dev 27: 2192-2206, 2013.

2. Larue L and Bellacosa A: Epithelial-mesenchymal transition in development and cancer: role of phosphatidylinositol 3' kinase/ AKT pathways. Oncogene 24: 7443-7454, 2005.

3. Thompson EW, Newgreen DF and Tarin D: Carcinoma invasion and metastasis: a role for epithelial-mesenchymal transition? Cancer Res 65: 5991-5995, 2005.

4. Hugo H, Ackland ML, Blick T, Lawrence MG, Clements JA, Williams ED and Thompson EW: Epithelial-mesenchymal and mesenchymal-epithelial transitions in carcinoma progression. $\mathrm{J}$ Cell Physiol 213: 374-383, 2007.

5. Polyak K and Weinberg RA: Transitions between epithelial and mesenchymal states: acquisition of malignant and stem cell traits. Nat Rev Cancer 9: 265-273, 2009.

6. Singh A and Settleman J: EMT, cancer stem cells and drug resistance: an emerging axis of evil in the war on cancer. Oncogene 29: 4741-4751, 2010.

7. Thiery JP and Sleeman JP: Complex networks orchestrate epithelial-mesenchymal transitions. Nat Rev Mol Cell Biol 7: 131-142, 2006

8. Christofori G: New signals from the invasive front. Nature 441: 444-450, 2006.

9. Bronner C: Control of DNMT1 abundance in epigenetic inheritance by acetylation, ubiquitylation, and the histone code. Sci Signal 4: pe3, 2011.

10. Hashimoto H, Vertino PM and Cheng X: Molecular coupling of DNA methylation and histone methylation. Epigenomics 2: 657-669, 2010

11. Du Z, Song J, Wang Y, et al: DNMT1 stability is regulated by proteins coordinating deubiquitination and acetylation-driven ubiquitination. Sci Signal 3: ra80, 2010.

12. Hong $Q$ and Shao ZM: Ubiquitination/deubiquitination and acetylation/deacetylation: making DNMT1 stability more coordinated. Acta Pharmacol Sin 32: 139-140, 2011.
13. Bronner C, Krifa M and Mousli M: Increasing role of UHRF1 in the reading and inheritance of the epigenetic code as well as in tumorogenesis. Biochem Pharmacol 86: 1643-1649, 2013.

14. Pacaud R, Brocard E, Lalier L, Hervouet E, Vallette FM and Cartron PF: The DNMT1/PCNA/UHRF1 disruption induces tumorigenesis characterized by similar genetic and epigenetic signatures. Sci Rep 4: 4230, 2014.

15. Hervouet E, Lalier L, Debien E, et al: Disruption of Dnmt1/ PCNA/UHRF1 interactions promotes tumorigenesis from human and mice glial cells. PLoS One 5: e11333, 2010.

16. Livak KJ and Schmittgen TD: Analysis of relative gene expression data using real-time quantitative PCR and the 2(-Delta Delta C(T)) method. Methods 25: 402-408, 2001

17. Scheel C and Weinberg RA: Cancer stem cells and epithelialmesenchymal transition: concepts and molecular links. Semin Cancer Biol 22: 396-403, 2012.

18. Morel AP, Lievre M, Thomas C, Hinkal G, Ansieau S and Puisieux A: Generation of breast cancer stem cells through epithelial-mesenchymal transition. PLoS One 3: e2888, 2008.

19. Jung MJ, Rho JK, Kim YM, et al: Upregulation of CXCR4 is functionally crucial for maintenance of stemness in drugresistant non-small cell lung cancer cells. Oncogene 32: 209-221, 2013.

20. Korkaya H, Kim GI, Davis A, et al: Activation of an IL6 inflammatory loop mediates trastuzumab resistance in HER $2^{+}$breast cancer by expanding the cancer stem cell population. Mol Cell 47: 570-584, 2012.

21. Sukowati CH, Rosso N, Croce LS and Tiribelli C: Hepatic cancer stem cells and drug resistance: relevance in targeted therapies for hepatocellular carcinoma. World J Hepatol 2: 114-126, 2010.

22. Yang ZF, Ho DW, Ng MN, et al: Significance of CD90 ${ }^{+}$cancer stem cells in human liver cancer. Cancer Cell 13: 153-166, 2008.

23. Li X, Ma Q, Xu Q, et al: $\mathrm{SDF}-1 / \mathrm{CXCR} 4$ signaling induces pancreatic cancer cell invasion and epithelial-mesenchymal transition in vitro through non-canonical activation of Hedgehog pathway. Cancer Lett 322: 169-176, 2012.

24. Bertran E, Caja L, Navarro E, Sancho P, Mainez J, Murillo MM, Vinyals A, Fabra A and Fabregat I: Role of CXCR4/SDF-1 alpha in the migratory phenotype of hepatoma cells that have undergone epithelial-mesenchymal transition in response to the transforming growth factor-beta. Cell Signal 21: 1595-1606, 2009.

25. Wan L, Pantel K and Kang Y: Tumor metastasis: moving new biological insights into the clinic. Nat Med 19: 1450-1464, 2013.

26. Tam WL and Weinberg RA: The epigenetics of epithelialmesenchymal plasticity in cancer. Nat Med 19: 1438-1449, 2013.

27. Chu J, Loughlin EA, Gaur NA, et al: UHRF1 phosphorylation by cyclin A2/cyclin-dependent kinase 2 is required for zebrafish embryogenesis. Mol Biol Cell 23: 59-70, 2012.

28. Sabatino L, Fucci A, Pancione M, et al: UHRF1 coordinates peroxisome proliferator activated receptor gamma (PPARG) epigenetic silencing and mediates colorectal cancer progression. Oncogene 31: 5061-5072, 2012.

29. Wang F, Yang YZ, Shi CZ, Zhang P, Moyer MP, Zhang HZ, Zou Y and Qin HL: UHRF1 promotes cell growth and metastasis through repression of $16^{\text {ink4a }}$ in colorectal cancer. Ann Surg Oncol 19: 2753-2762, 2012.

30. Zhou L, Zhao X, Han Y, et al: Regulation of UHRF1 by miR$146 \mathrm{a} / \mathrm{b}$ modulates gastric cancer invasion and metastasis. FASEB J 27: 4929-4939, 2013

31. Li XL, Xu JH, Nie JH and Fan SJ: Exogenous expression of UHRF1 promotes proliferation and metastasis of breast cancer cells. Oncol Rep 28: 375-383, 2012.

32. Mudbhary R, Hoshida Y, Chernyavskaya Y, et al: UHRF1 overexpression drives DNA hypomethylation and hepatocellular carcinoma. Cancer Cell 25: 196-209, 2014.

33. Karpf AR and Matsui S: Genetic disruption of cytosine DNA methyltransferase enzymes induces chromosomal instability in human cancer cells. Cancer Res 65: 8635-8639, 2005.

34. Liu S, Liu Z, Xie Z, et al: Bortezomib induces DNA hypomethylation and silenced gene transcription by interfering with Sp1/NF-kappaB-dependent DNA methyltransferase activity in acute myeloid leukemia. Blood 111: 2364-2373, 2008.

35. Batlle E, Sancho E, Franci C, Dominguez D, Monfar M, Baulida J and Garcia De Herreros A: The transcription factor snail is a repressor of E-cadherin gene expression in epithelial tumour cells. Nat Cell Biol 2: 84-89, 2000. 
36. Cano A, Perez-Moreno MA, Rodrigo I, Locascio A, Blanco MJ, del Barrio MG, Portillo F and Nieto MA: The transcription factor snail controls epithelial-mesenchymal transitions by repressing E-cadherin expression. Nat Cell Biol 2: 76-83, 2000.

37. Guaita S, Puig I, Franci C, et al: Snail induction of epithelial to mesenchymal transition in tumor cells is accompanied by MUC1 repression and ZEB1 expression. J Biol Chem 277: 39209-39216, 2002.

38. Darash-Yahana M, Pikarsky E, Abramovitch R, et al: Role of high expression levels of CXCR4 in tumor growth, vascularization, and metastasis. FASEB J 18: 1240-1242, 2004.

39. Scala S, Giuliano P, Ascierto PA, et al: Human melanoma metastases express functional CXCR4. Clin Cancer Res 12: 2427-2433, 2006.

40. Wald O, Shapira OM and Izhar U: CXCR4/CXCL12 axis in non small cell lung cancer (NSCLC) pathologic roles and therapeutic potential. Theranostics 3: 26-33, 2013.

41. Su L, Zhang J, Xu H, Wang Y, Chu Y, Liu R and Xiong S: Differential expression of CXCR4 is associated with the metastatic potential of human non-small cell lung cancer cells. Clin Cancer Res 11: 8273-8280, 2005.
42. Dubrovska A, Hartung A, Bouchez LC, Walker JR, Reddy VA, Cho CY and Schultz PG: CXCR4 activation maintains a stem cell population in tamoxifen-resistant breast cancer cells through AhR signalling. Br J Cancer 107: 43-52, 2012.

43. Gelmini S, Mangoni M, Serio M, Romagnani P and Lazzeri E: The critical role of SDF-1/CXCR4 axis in cancer and cancer stem cells metastasis. J Endocrinol Invest 31: 809-819, 2008.

44. Miki J, Furusato B, Li H, et al: Identification of putative stem cell markers, CD133 and CXCR4, in hTERT-immortalized primary nonmalignant and malignant tumor-derived human prostate epithelial cell lines and in prostate cancer specimens. Cancer Res 67: 3153-3161, 2007.

45. Kim JK, Esteve PO, Jacobsen SE and Pradhan S: UHRF1 binds G9a and participates in p21 transcriptional regulation in mammalian cells. Nucleic Acids Res 37: 493-505, 2009. 